\title{
Producción científica sobre la simulación clínica: revisión integrativa de las tesis y disertaciones brasileñas
}

\author{
Scientific production on clinical simulation: \\ integrative review of Brazilian theses and dissertations
}

Saionara Nunes de Oliveira, * Jussara Gue Martini, ** Jaime Alonso Caravaca-Morera***

\section{Palabras clave: Simulación, disertaciones académicas, revisión, Brasil.}

Key words: Simulation, academic dissertations, review, Brazil.

\section{RESUMEN}

Introducción: La investigación sobre simulación clínica en Brasil es reciente y está en expansivo crecimiento. Conocer las investigaciones que se están desarrollando en esta área es de extrema importancia para los estudiosos del tema. Objetivo: Caracterizar las investigaciones sobre simulación clínica desarrolladas en las tesis y disertaciones realizadas en Brasil. Método: Revisión integrativa realizada en el Catálogo de Tesis y Disertaciones de la CAPES (Coordinación de Mejora de Personal de Nivel Superior). La búsqueda ocurrió en agosto de 2018 utilizando las expresiones "Simulación Clínica” OR “Simulación Realista”. Resultados: Se identificaron 28 disertaciones de maestría $(65.1 \%)$ y 15 tesis de doctorado (34.9\%) concluidas entre 2010 y 2017 , predominantes de las regiones sudeste y nordeste $(86 \%)$, provenientes de programas de postgrado en enfermería $(62.8 \%)$, con delineamiento investigativo cuantitativo $(69.81 \%)$ e investigando principalmente el aprendizaje proporcionado por la simulación para determinados contenidos (37.2\%). Conclusión: La investigación sobre simulación clínica en Brasil es reciente y se concentra en evaluar la eficacia del método en la retención de contenidos específicos. La enfermería está liderando las investigaciones sobre simulación y el abordaje cualitativo es incipiente. Poco se ha discutido sobre la parte pedagógica del método y no aparecen investigaciones sobre la interdisciplinaridad.

\section{ABSTRACT}

Introduction: Research on clinical simulation in Brazil is recent and is expanding. To know the researches that are being developed in this area is of extreme importance for scholars of the subject. Objective: To characterize the researches on clinical simulation developed in theses and dissertations carried out in Brazil. Method: Integrative revision made in the Catalog of Thesis and Dissertations of CAPES (Coordination of Superior Level Staff Improvement). The search occurred in August 2018 using the expressions "clinical simulation" $O R$ "realistic simulation”. Results: 28 master's dissertations (65.1\%) and 15 doctoral theses (34.9\%) were completed between 2010 and 2017, predominantly from the southeastern and northeastern regions (86\%), from nursing postgraduate program (62.8\%), with a quantitative research design (69.81\%) and mainly investigating the learning provided by the simulation for certain contents (37.2\%). Conclusion: Research on clinical simulation in Brazil is recent and focuses on evaluating the efficacy of the method in the retention of specific contents. Nursing is leading the research on simulation and the qualitative approach is incipient. Little has been discussed about the pedagogical part of the method, just as there are no investigations about interdisciplinarity.

\section{INTRODUCCIÓN}

T a simulación es una práctica que fue im$ـ$ pulsada y ganó notoriedad después de la Segunda Guerra Mundial. Fue utilizada por la aviación en el entrenamiento de pilotos para el enfrentamiento de adversidades climáticas, averías en pleno vuelo y para mejorar las competencias de un modo general. ${ }^{1}$

En la salud, la simulación ganó visibilidad a partir de la década de 1960, con la creación de los simuladores para entrenamiento médico en reanimación cardiopulmonar "Resusci Anne" y auscultación cardiaca "Harvey". 2,3

Sin embargo, la enfermería ya utilizaba simuladores de baja fidelidad al inicio del siglo XX. Hay registro del simulador "Mrs. Chase" en 1910. ${ }^{4}$ Este simulador fue encomendado a una empresa de juguetes por una enfermera de la Escuela de Formación de Enfermeras del Hospital Hartford, ubicado en Connecticut, Estados Unidos de América. Era utilizado para el entrenamiento de diversas habilidades básicas de la enfermería y tenía la ventaja de ser construido 
con material resistente, lo que permitía mayor durabilidad, diferente de los modelos antiguos construidos con paja. Como podemos ver, el uso de simuladores por la enfermería es más antiguo de lo que tenemos registro. ${ }^{5}$

Los simuladores fueron ganando espacio en los diferentes cursos del área de la salud, en los más distintos niveles de fidelidad.

En la enfermería brasileña no es reciente el uso de estrategias simuladas: hay registro del uso de un simulador de baja fidelidad (maniquí) desde $1920,{ }^{6}$ y a lo largo de los años se incorporaron otros tipos de simuladores utilizados para el entrenamiento de las más variadas habilidades: punción venosa, reanimación cardiorrespiratoria, auscultación pulmonar, etcétera. Sin embargo, todo eso puede ser clasificado como entrenamiento de habilidades, que es una importante estrategia y parte esencial en el desarrollo de competencias clínicas, pero solo no representa la simulación clínica.

La simulación clínica fue definida en un estudio que analizó los diferentes conceptos atribuidos a ella como:

"[...] un proceso dinámico que involucra la creación de una oportunidad hipotética que incorpora una representación auténtica de la realidad, facilitando la participación activa del alumno e integrando las complejidades del aprendizaje práctico y teórico con oportunidades para la repetición, retroalimentación, evaluación y reflexión." ${ }^{7}$

En este sentido, la simulación clínica no se limita al uso de un simulador. Es una estrategia de enseñanza que necesita de planificación pedagógica con objetivos claros de aprendizaje, una técnica apropiada de desarrollo, recursos físicos, humanos y materiales. ${ }^{8}$ Tiene como principal diferencia del entrenamiento de habilidades el momento de reflexión denominado debriefing (informe), con enfoque de la acción en la reflexión del estudiante mediada por el facilitador.

La simulación permite evaluar indicadores de competencias que son elementos importantes en el acompañamiento pedagógico de los estudiantes; sin embargo, la competencia clínica en general solamente se puede evaluar por el profesor en el acompañamiento del estudiante que realiza una atención real, ya que está compuesta de varios elementos que se articulan en la complejidad del cuidado. ${ }^{9}$ Por eso, la simulación no debe reemplazar el contacto real con los pacientes, sino anteceder este contacto para preparar mejor al estudiante para una práctica segura. ${ }^{10}$

Los centros de simulación clínica en Brasil se están expandiendo rápidamente y tienden a crecer, considerando la existencia de un expresivo número de cursos superiores presenciales del área de la salud en actividad: 5,880 según datos del E-MEC (2018).

Eso justifica la creciente búsqueda en los últimos años de espacios de discusión sobre el uso de la simulación clínica en los cursos del área de la salud, hecho observado en distintos eventos científicos realizados en el país, con demanda cada vez mayor de profesores y estudiantes de postgrado.

En este sentido, buscamos caracterizar las tesis y disertaciones producidas en Brasil a través de esta revisión integrativa.

\section{MÉTODO}

Se trata de una revisión integrativa de la literatura. Este tipo de estudio tiene por objetivo reunir información de varias investigaciones que puedan contribuir en la toma de decisiones, posibilitar la síntesis del conocimiento de determinado asunto y apuntar lagunas. Se siguieron los seis pasos de la revisión integrativa: ${ }^{11}$

1. Identificación del tema y selección de la hipótesis o cuestión de investigación para la elaboración de la revisión integrativa.

2. Establecimiento de criterios para la inclusión y exclusión de estudios/muestreo o consulta a la literatura.

3. Definición de las informaciones que serán extraídas de los estudios seleccionados/ categorización de los estudios.

4. Evaluación de los estudios incluidos en la revisión integrativa.

5. Interpretación de los resultados.

6. Presentación de la revisión/síntesis del conocimiento.

El desarrollo de esta revisión se justifica por la reciente inserción de Brasil en los estudios sobre simulación y los relatos de las experiencias iniciales del uso de la estrategia de enseñanza 
Tabla 1. Disertaciones brasileñas acerca de la simulación clínica/simulación realista.

\begin{tabular}{|c|c|c|c|}
\hline Código & Año & Autor & Título \\
\hline M1 & 2010 & Santos, Mateus Casanova Dos & $\begin{array}{l}\text { Estudio de la evaluación en el disparador de aprendizaje simulación- } \\
\text { laboratorio morfofuncional/Facultad de Enfermería UFPEL }\end{array}$ \\
\hline M2 & 2013 & Oliveira, Saionara Nunes De & $\begin{array}{l}\text { Simulación clínica con participación de actores en la enseñanza de la } \\
\text { consulta de enfermería: una investigación-acción }\end{array}$ \\
\hline M3 & 2014 & $\begin{array}{l}\text { Costa, Raphael Raniere } \\
\text { De Oliveira }\end{array}$ & $\begin{array}{l}\text { La simulación realista como estrategia de enseñanza-aprendizaje en } \\
\text { enfermería }\end{array}$ \\
\hline M4 & 2014 & Moura, Luciana Carvalho & $\begin{array}{l}\text { Resultados de un programa de capacitación en donación de órganos para } \\
\text { trasplantes por medio de la simulación realista: impacto del entrenamiento } \\
\text { en los indicadores del proceso de donación de órganos }\end{array}$ \\
\hline M5 & 2014 & Couto, Thomaz Bittencourt & $\begin{array}{l}\text { Simulación realista en la enseñanza de emergencias pediátricas en la } \\
\text { graduación }\end{array}$ \\
\hline M6 & 2015 & $\begin{array}{l}\text { Sousa, Jairo Edielson Rodrigues } \\
\text { Barbosa De }\end{array}$ & $\begin{array}{l}\text { Experiencia de simulación clínica avanzada en enfermería: satisfacción y } \\
\text { autoconfianza como resultados }\end{array}$ \\
\hline M7 & 2015 & Brito, Paulo Santiago De Morais & $\begin{array}{l}\text { Simulación como método de enseñanza de habilidades en la carrera de } \\
\text { Medicina: relato de una experiencia con punción lumbar }\end{array}$ \\
\hline M8 & 2015 & Fabri, Renata Paula & Construcción de pasos teórico-prácticos para la actividad simulada \\
\hline M9 & 2016 & Lopes, Vanessa Caminha Aguiar & $\begin{array}{l}\text { Medida del componente conocimiento de la competencia “evaluación } \\
\text { de riesgo para lesión por presión de Moura”: calidad psicométrica por } \\
\text { simulación avanzada }\end{array}$ \\
\hline M10 & 2016 & $\begin{array}{l}\text { Nascimento, Priscyla } \\
\text { De Oliveira }\end{array}$ & $\begin{array}{l}\text { Construcción y validación del escenario de simulación clínica en el } \\
\text { manejo de la hemorragia postparto }\end{array}$ \\
\hline M11 & 2016 & Soares, Samuel Freitas & $\begin{array}{l}\text { Gestión del cuidado y simulación clínica: desarrollando la competencia } \\
\text { "comunicación” en enfermería }\end{array}$ \\
\hline M12 & 2016 & Santos, Claudenice Ferreira Dos & $\begin{array}{l}\text { Simulación realista como estrategia innovadora para la relación } \\
\text { enseñanza-aprendizaje en la enseñanza superior }\end{array}$ \\
\hline M13 & 2016 & Gleke, Manoela Machado & $\begin{array}{l}\text { Evaluación de la capacitación del equipo de enfermería para el cuidado } \\
\text { con catéter enteral }\end{array}$ \\
\hline M14 & 2016 & Ferreira, Raina Pleis Neves & $\begin{array}{l}\text { Uso de diferentes estrategias de enseñanza-aprendizaje de estudiantes del } \\
\text { área de la salud }\end{array}$ \\
\hline M15 & 2016 & Turole, Daniela Cristina Sandy & $\begin{array}{l}\text { Simulación realista como recurso metodológico en la enseñanza de } \\
\text { graduación en enfermería: percepción del alumno }\end{array}$ \\
\hline M16 & 2016 & Ferreira Filho, Francisco & $\begin{array}{l}\text { Modelo de enseñanza basada en simulación realista para la progresión de } \\
\text { habilidades en endosuturas en la cirugía mínimamente invasiva }\end{array}$ \\
\hline M17 & 2017 & Espadaro, Renato Fabio & $\begin{array}{l}\text { Simulación realista en la formación en enfermería: percepción de } \\
\text { docentes y discentes }\end{array}$ \\
\hline M18 & 2017 & Brasil, Guilherme Da Costa & Simulación realista como estrategia de enseñanza en enfermería materno infantil \\
\hline M19 & 2017 & $\begin{array}{l}\text { Oliveira, Danielle Leite } \\
\text { De Lemos }\end{array}$ & $\begin{array}{l}\text { Curso para capacitación de instructores de simulación clínica en } \\
\text { enfermería con uso de ambiente virtual de aprendizaje }\end{array}$ \\
\hline M20 & 2017 & David, Flavio Sampaio & $\begin{array}{l}\text { El método de la simulación realista en el área de urgencia y emergencia } \\
\text { en la construcción de la autonomía del estudiante en la enseñanza de } \\
\text { graduación en enfermería }\end{array}$ \\
\hline M21 & 2017 & Dos Reis, Nelida Beatriz Caldas & Adaptación cultural de la herramienta Health Communication Assessment Tool \\
\hline M22 & 2017 & Oliveira, Karillucy Mendes De & $\begin{array}{l}\text { Implementación de la simulación realista como método de mejora de la } \\
\text { seguridad del paciente: ensayo controlado }\end{array}$ \\
\hline M23 & 2017 & Silva, Daniele Carvalho Da & $\begin{array}{l}\text { Simulación realista como estrategia para el aprendizaje de la } \\
\text { comunicación del equipo multiprofesional }\end{array}$ \\
\hline
\end{tabular}


Continúa la Tabla 1. Disertaciones brasileñas acerca de la simulación clínica/simulación realista.

\begin{tabular}{|c|c|c|c|}
\hline Código & Año & Autor & Título \\
\hline M24 & 2017 & $\begin{array}{l}\text { Pinheiro, Eudes } \\
\text { Fontenele Moraes }\end{array}$ & $\begin{array}{l}\text { Modelo de entrenamiento con simulación realista para anastomosis vesi- } \\
\text { couretral laparoscópica }\end{array}$ \\
\hline M25 & 2017 & Menes, Ana Paula Borges & $\begin{array}{l}\text { La comunicación efectiva como premisa para la aplicación del Término de } \\
\text { Consentimiento Libre y Aclarado en participantes de investigación }\end{array}$ \\
\hline M26 & 2017 & Boostel, Radames & $\begin{array}{l}\text { Efecto de la simulación clínica de alta fidelidad en la ansiedad y en el } \\
\text { estrés del discente de graduación en enfermería }\end{array}$ \\
\hline M27 & 2017 & $\begin{array}{l}\text { Rodrigues, Heitor Hermeson } \\
\text { De Carvalho }\end{array}$ & $\begin{array}{l}\text { Evaluación de recursos tecnológicos de ingeniería biomédica de un simulador } \\
\text { humano computarizado: estudio de caso con simulación realista de la presión } \\
\text { arterial }\end{array}$ \\
\hline M28 & 2017 & Barroso, Karine Sampaio Nunes & $\begin{array}{l}\text { Simulación realista en el entrenamiento de estudiantes de medicina en } \\
\text { terapia transfusional: elaboración y aplicabilidad }\end{array}$ \\
\hline
\end{tabular}

Fuente: Datos de la investigación.

por instituciones brasileñas en los eventos internacionales sobre el tema. En este sentido, el estudio tiene por cuestión orientadora: ¿qué ha sido investigado sobre simulación clínica en las tesis y disertaciones desarrolladas en Brasil? Y como objetivo: caracterizar las investigaciones sobre simulación clínica desarrolladas en las tesis y disertaciones realizadas en Brasil.

Se optó por investigar las producciones de las tesis y disertaciones por entender que este tipo de investigación recibe más estímulo y fomento, además de mayor tiempo para su realización, lo que permite estudios más profundizados. Se utilizó como base de datos el Catálogo de Tesis y Disertaciones de la Coordinación de Perfeccionamiento de Personal de Nivel Superior (CAPES) del Ministerio de Educación (MEC), que es parte del Portal de Periódicos de CAPES/MEC, donde es posible consultar los resúmenes de las tesis y disertaciones defendidas a partir de 1987. En este portal, las informaciones son proporcionadas de manera directa por los programas de postgrado, que se responsabilizan por la veracidad de los datos.

Se aplicaron como criterios de inclusión: estudios disponibles en línea recuperados a partir de la clave de búsqueda "simulación clínica" OR "simulación realista" (términos usualmente empleados en Brasil como sinónimos); y como criterios de exclusión: estudios en que la simulación aparecía sólo como resultado, estudios de otras áreas que no fueran de salud, estudios que no abordaran la simulación clínica.

Esta etapa fue realizada en el mes de agosto de 2018 y recuperó 76 trabajos; 33 de ellos fueron excluidos después de la aplicación de los criterios de exclusión. Tras la selección de los 43 estudios, se realizó la lectura detallada de los trabajos, primeramente el título y resumen, y después, en su totalidad, con el objetivo de identificar toda la información necesaria.

Fue extraída la siguiente información: nombre del autor, profesión, año de publicación, programa de postgrado, institución de enseñanza superior, carrera, título del estudio, objetivo, método, participantes y conclusiones. Esa información fue organizada en una planilla electrónica y analizada confrontando los hallazgos con la literatura.

Fueron atribuidos códigos alfanuméricos para identificar cada uno de los estudios y para referirlos durante la discusión. Se utilizó la letra M para disertaciones de máster y $\mathrm{D}$ para tesis de doctorado, seguidos de números según el orden cronológico del trabajo.

La síntesis del conocimiento se está presentando en este artículo científico.

\section{RESULTADOS}

Del total de 43 trabajos seleccionados para análisis, 28 fueron disertaciones de máster (Tabla 1) y 15 tesis de doctorado (Tabla 2). Los 
Tabla 2. Tesis brasileñas acerca de la simulación clínica/simulación realista.

\begin{tabular}{|c|c|c|c|}
\hline Código & Año & Autor & Título \\
\hline D1 & 2013 & $\begin{array}{l}\text { Moura, Elaine Cristina } \\
\text { Carvalho }\end{array}$ & $\begin{array}{l}\text { Enseñanza-aprendizaje de enfermería en simulación clínica: desarrollando } \\
\text { competencia profesional para la prevención de úlceras por presión }\end{array}$ \\
\hline D2 & 2014 & Schweller, Marcelo & La enseñanza de empatía en el curso de graduación en Medicina \\
\hline D3 & 2014 & $\begin{array}{l}\text { Grassia, Rita De Cassia } \\
\text { Fernandes }\end{array}$ & $\begin{array}{l}\text { Evaluación del aprendizaje del electrocardiograma por el método tradicional } \\
\text { y en el centro de simulación realista }\end{array}$ \\
\hline D4 & 2014 & Machado, Juliana Pereira & $\begin{array}{l}\text { Intervención educativa sobre la medida indirecta de la presión arterial por } \\
\text { profesionales de enfermería: una propuesta para la seguridad del paciente }\end{array}$ \\
\hline D5 & 2015 & $\begin{array}{l}\text { Junior, Luiz Gonzaga } \\
\text { De Moura }\end{array}$ & $\begin{array}{l}\text { Modelo académico de enseñanza teórico-práctica en videocirugía por } \\
\text { medio de nuevo simulador real de cavidad abdominal }\end{array}$ \\
\hline D6 & 2015 & Quilici, Ana Paula & $\begin{array}{l}\text { La vivencia de los docentes en la simulación clínica insertada en el } \\
\text { currículo: desafíos, dificultades y conquistas }\end{array}$ \\
\hline D7 & 2015 & Vilela, Danielle Monteiro & $\begin{array}{l}\text { La enseñanza de la evaluación clínica de la oxigenación y circulación del } \\
\text { bebé pretérmino: integración, simulación virtual y simulación robótica }\end{array}$ \\
\hline D8 & 2015 & Soares, Carolina Felipe & $\begin{array}{l}\text { El uso de la simulación realista de alta fidelidad como estrategia } \\
\text { educacional: evaluación del aprendizaje procedimental en emergencia } \\
\text { cardiovascular }\end{array}$ \\
\hline D9 & 2016 & $\begin{array}{l}\text { Paula, Maria De Fatima } \\
\text { Correa }\end{array}$ & $\begin{array}{l}\text { Simulación realista sobre delirium en adultos mayores: conocimiento, } \\
\text { satisfacción y autoconfianza en el aprendizaje }\end{array}$ \\
\hline D10 & 2016 & Perbone, Janaina Gomes & $\begin{array}{l}\text { Juzgamiento clínico y raciocinio diagnóstico de estudiantes de enfermería } \\
\text { en simulación clínica de alta fidelidad }\end{array}$ \\
\hline D11 & 2016 & $\begin{array}{l}\text { Almeida, Rodrigo Guimaraes } \\
\text { Dos Santos }\end{array}$ & $\begin{array}{l}\text { Simulación clínica: validación de instrumentos de enseñanza-aprendizaje } \\
\text { para la lengua portuguesa }\end{array}$ \\
\hline D12 & 2016 & $\begin{array}{l}\text { Canova, Jocilene De Carvalho } \\
\text { Miraveti }\end{array}$ & Soporte básico de vida para no profesionales: un estudio casi experimental \\
\hline D13 & 2017 & $\begin{array}{l}\text { Janicas, Rita De Cassia } \\
\text { Silva Vieira }\end{array}$ & $\begin{array}{l}\text { Contribución de los escenarios debriefing en el proceso enseñanza- } \\
\text { aprendizaje de graduandos de enfermería }\end{array}$ \\
\hline D14 & 2017 & $\begin{array}{l}\text { Rodrigues, Iellen Dantas } \\
\text { Campos Verdes }\end{array}$ & $\begin{array}{l}\text { Simulación realista en el proceso de enseñanza-aprendizaje del racioci- } \\
\text { nio diagnóstico de enfermería }\end{array}$ \\
\hline D15 & 2017 & Tiburcio, Manuela Pinto & $\begin{array}{l}\text { Simulación realista como estrategia de enseñanza-aprendizaje en el } \\
\text { proceso transfusional }\end{array}$ \\
\hline
\end{tabular}

Fuente: Datos de la investigación.

trabajos fueron presentados en el periodo de 2010 a 2017, según la figura 1 .

Los trabajos se desarrollaron en instituciones de enseñanza públicas y privadas, siendo predominantes las instituciones públicas federales. Estuvieron concentrados en las regiones sudeste y nordeste; solamente la región norte no tuvo tesis y disertaciones sobre la temática. La tabla 3 presenta el nombre de las instituciones y su respectiva sigla, la naturaleza, región y el número referente de trabajos vinculados a cada una de ellas.
En cuanto a la distribución de acuerdo con el área del programa de postgrado, se nota, a través de los datos presentados en la figura 2, el predominio en el área de enfermería.

Los investigadores fueron en su mayoría enfermeros, 34 (79.1\%); seguidos de médicos, siete $(16.3 \%)$; un ingeniero electrónico $(2.3 \%$ ) y un biomédico (2.3\%). En cuanto al tipo de estudio, la mayoría utilizó el método cuantitativo, 30 (69.8\%), seguido del método cualitativo, seis (14\%); método cuantitativo-cualitativo, cinco (11.6\%), y estudios metodológicos, dos (4.6\%). 
Los sujetos investigados están presentados en la tabla 4 y son en su mayoría estudiantes (graduación, especialización, nivel técnico y residencia); aparecieron también estudios con

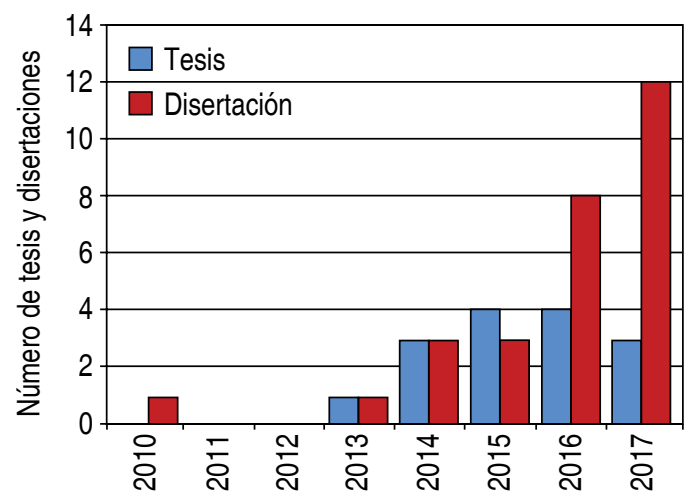

Figura 1. Distribución de tesis y disertaciones al año. profesores, aunque en menor cantidad; también especialistas, egresados y profesionales.

Los estudios tuvieron como principal objetivo verificar el aprendizaje proporcionado por la simulación en temas específicos. Los temas utilizados en los trabajos que buscaban evaluar el aprendizaje de determinados contenidos fueron: evaluación de riesgo para úlceras por presión, consulta de enfermería, punción lumbar, oxigenación y circulación del bebé pretérmino, emergencia cardiovascular, catéter enteral, comunicación profesional, juzgamiento clínico y raciocinio diagnóstico, delirio en adultos mayores, reanimación cardiopulmonar, proceso transfusional, presión arterial, efectiva comunicación para la aplicación del Término de Consentimiento Libre y Aclarado, empatía (M2; M7; M9; M11; M13; M25; M27; D1; D2; D4; D7; D8; D9; D10; D14; D15).

\section{Tabla 3. Distribución de los trabajos por institución de enseñanza, naturaleza y región de Brasil.}

\begin{tabular}{lcccc} 
& Sigla & Naturaleza & $\begin{array}{c}\text { Región de } \\
\text { Brasil }\end{array}$ & $\begin{array}{c}\text { Número de } \\
\text { trabajos }\end{array}$ \\
\hline Nombre de la institución & USP & Pública/Estatal & Sudeste & 11 \\
Universidad de São Paulo & UFRN & Pública/Federal & Nordeste & 4 \\
Universidad Federal de Río Grande do Norte & UNIFESP & Pública/Federal & Sudeste & 4 \\
Universidad Federal de São Paulo & UFPI & Pública/Federal & Nordeste & 3 \\
Universidad Federal de Piaú & UNICHRISTUS & Privada/Con ánimo de lucro & Nordeste & 3 \\
Centro Universitario Christus & UNICAMP & Pública/Estatal & Sudeste & 3 \\
Universidad Estadual de Campinas & UNB & Pública/Federal & Centro-oeste & 2 \\
Universidad de Brasilia & USP & Pública/Estatal & Sudeste & 2 \\
Universidad de São Paulo & UFC & Pública/Federal & Nordeste & 1 \\
Universidad Federal de Ceará & UFPE & Pública/Federal & Nordeste & 1 \\
Universidad Federal de Pernambuco & UNEB & Pública/Estatal & Nordeste & 1 \\
Universidad del Estado de Bahía & PUC & Privada & Sudeste & 1 \\
Pontificia Universidad Católica de São Paulo & FICSAE & Privada/Sin ánimo de lucro & Sudeste & 1 \\
Facultad Israelita de Ciencias de la Salud & & & Sudeste & 1 \\
Albert Einstein & UMC & Privada/con ánimo de lucro & Sudeste & 1 \\
Universidad de Mogi das Cruzes & UFRJ & Pública/Federal & Sudeste & 1 \\
Universidad Federal de Río de Janeiro & UFVJM & Pública/Federal & & \\
Universidad Federal de Vales do Jequitinhon- & & & Sudeste & 1 \\
ha y Mucuri & UNINOVE & Privada/sin ánimo de lucro & Sul & 1 \\
Universidad Nove de Julho & UFPEL & Pública/Federal & Sul & 1 \\
Universidad Federal de Pelotas & UFSC & Pública/Federal & Sul & 1 \\
Universidad Federal de Santa Catarina & UFPR & Pública/Federal & Sul & 1 \\
Universidad Federal de Paraná & UTFPR & Pública/Federal & & \\
Universidad Tecnológica Federal do Paraná & & & & \\
\hline Fuente: Datos de la investigación. & & & & \\
& & & & \\
\end{tabular}


Esos estudios señalan la eficacia de la simulación como estrategia de enseñanza para todas esas temáticas, además de rescatar el raciocinio operativo de los estudiantes durante la acción, desarrollar el pensamiento crítico-reflexivo sobre la competencia, identificar lagunas de aprendizaje, promover la satisfacción de los estudiantes y mejorar la autoimagen profesional, movilizar a los alumnos hacia un protagonismo en búsqueda del conocimiento, contribuir para mejorar el conocimiento, la satisfacción y la autoconfianza en el aprendizaje.
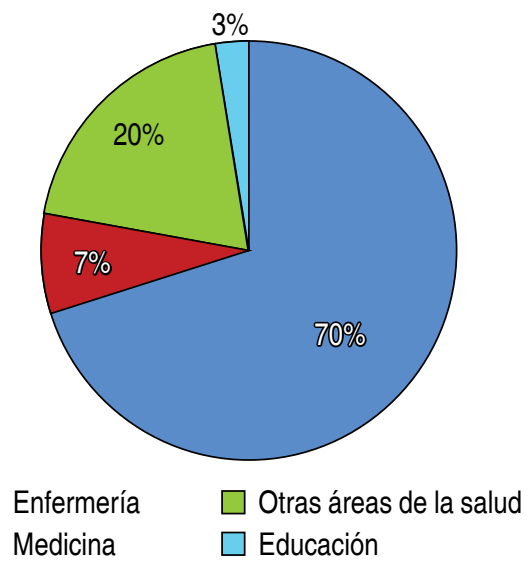

Figura 2. Distribución de los trabajos por área de los programas de postgrado.
Algunos trabajos buscaron comprender el significado de la simulación para profesores (D6), estudiantes (M14; M15; M6) y/o profesionales (M23). En ellos, el énfasis era en cuanto a desafíos, dificultades y conquistas en la aplicación de la simulación por los profesores; conocimiento, satisfacción y autoconfianza de los alumnos; proceso de enseñanza y aprendizaje; evaluación. Los resultados demuestran que los profesores notaron una necesidad de adecuación logística para que pudieran utilizar mejor la propuesta de la simulación, el número de estudiantes por docente, el tiempo exigido para la elaboración de los escenarios y la necesidad de entrenamiento continuo de los docentes. Los estudiantes evalúan positivamente la estrategia y presentan mejora en los índices de satisfacción y autoconfianza en el aprendizaje. La simulación favorece el desarrollo del pensamiento crítico y reflexivo en los estudiantes. Los profesionales también son beneficiados por la simulación y pueden ejercitar habilidades de comunicación y relación con el paciente en situaciones de conflictos y quejas.

En los estudios que trataban de instrumentos y pasos, las temáticas fueron validación de escenario, traducción y validación a la lengua portuguesa de escalas americanas, propuesta de modelo para simulación, capacitación docente (M10; M19; M21; M28; D11).

Tabla 4. Sujetos investigados en los estudios.

\begin{tabular}{ll} 
Sujetos & Estudios \\
\hline Estudiantes de graduación & M2; M5; M7; M9; M12; M14; M15; M18; M22; \\
& M26; D2; D10; D12; D13 \\
Estudiantes de postgrado & M25; D3; D9 \\
Estudiantes de nivel técnico & M27 \\
Especialistas & M8 \\
Profesores & M19; D6 \\
Profesionales & M4; M13; M23; D4; D11; D15 \\
Estudiantes de graduación y especialistas & M10; M11; D7; D14 \\
Estudiantes de graduación y profesores & M1; M3; M17; M20; D1; D8 \\
Estudiantes de graduación y egresados & M6 \\
Residentes y especialistas & M24; M16 \\
Estudiantes, residentes y profesionales & D5 \\
Estudiantes, profesionales y especialistas & M28 \\
\hline Fuente: Datos de la investigación. &
\end{tabular}


Un escenario para el manejo de la hemorragia postparto fue propuesto en uno de los estudios; en otro, fue desarrollado, implementado y evaluado un curso para la capacitación de instructores de simulación clínica en enfermería usando un ambiente virtual de aprendizaje (M10; M19).

Se realizó la adaptación cultural de la herramienta Health Communication Assessment Tool-HCAT al portugués brasileño, y podrá ser útil para evaluar la capacidad comunicativa de los estudiantes de enfermería durante escenarios de simulación clínica. Un método de entrenamiento en medicina transfusional fue desarrollado, con manual y escenario de simulación en salud. La versión en lengua portuguesa del examen de competencia en hemoterapia (BEST TEST) fue validada para uso en la población brasileña, así como cuatro escalas americanas: 1) student satisfaction and self-confidence in learning, 2) debriefing experience scale, 3) simulation design scale y 4) educational practices questionnaire (M21, M28, D11).

Los estudios que evaluaron la metodología de la simulación la identificaron como un mecanismo generador de expectativa y satisfacción entre los discentes, que influencia la calidad del aprendizaje de enfermería, especialmente en la formación consolidada en estándares de seguridad y eficiencia. Identificaron que la simulación puede contribuir para la enseñanza de enfermería favoreciendo el aprendizaje crítico y reflexivo, promoviendo la autoevaluación frente a la responsabilidad con el aprendizaje y con el paciente. Recomiendan considerar la percepción del participante en cuanto a su actuación en un escenario simulado y cómo consigue asimilar el escenario al cual es sometido. Sugieren el uso de pasos para aplicar la enseñanza simulada para facilitar el trabajo docente (M1; M3; M8; M17; M18; M20; M26).

La comparación de la simulación con otra estrategia apareció en seis estudios, cinco de ellos compararon la simulación con métodos tradicionales de enseñanza y uno comparó con y sin debriefing. Los temas trabajados fueron RCP, electrocardiograma, seguridad del paciente en la administración de medicamentos, emergencias pediátricas y vacunas. Esos estudios mostraron que la simulación tuvo re- sultados tan buenos o mejores que los métodos tradicionales, especialmente en aspectos como la satisfacción de los estudiantes, desarrollo de habilidades y retención de conocimientos. El debriefing se constituyó como estrategia facilitadora del proceso de enseñanza y aprendizaje (M5; M12; M22; D3; D12; D13).

Un trabajo buscó identificar el impacto de un programa de capacitación que utilizaba simulación en los indicadores de donación de órganos. Se puede notar que cuanto mayor era el número de profesionales capacitados, mayor fue la tasa de conversión, es decir, de potenciales donadores cuya familia autorizó la donación de órganos, y que cuanto mayor era la retención del conocimiento después del curso, menor fue la tasa de rechazo familiar (M4).

Otros tres estudios tuvieron por objetivo proponer modelos o simuladores para su uso en el área médica en videocirugías, y se mostraron efectivos en el desarrollo de estas habilidades (M16; M24; D5).

\section{DISCUSIÓN}

La enfermería lidera las encuestas sobre simulación clínica desarrolladas en Brasil en tesis y disertaciones. Este hecho puede estar relacionado con la larga trayectoria de la profesión en el uso de laboratorios de simulación como espacio de enseñanza y aprendizaje.

En Brasil, un estudio que trató de hacer un rescate histórico del uso de simuladores por la enfermería, encontró pocos trabajos nacionales publicados, a pesar de registros de su utilización desde la década de $1920 .^{6}$

La enfermería brasileña tuvo su primera escuela en 1923 en Río de Janeiro, siguiendo el Sistema Nightingale y orientado por enfermeras norteamericanas. La Escuela Anna Nery, desde su creación, ya contaba con laboratorios denominados "sala de clases prácticas" y utilizaba maniquís, siguiendo la influencia americana. Sin embargo, los simuladores que para la época eran la representación de la modernidad, siguieron siendo utilizados en las escuelas de enfermería brasileñas durante cerca de 70 años, sin acompañar la evolución tecnológica internacional. Pocos estudios se realizaron para evaluar esta herramienta educativa; solamente en 1990 se comenzaron a esbozar algunas crí- 
ticas relacionadas con la cantidad insuficiente de simuladores para el número de alumnos, y a partir de entonces, otros estudios críticos evaluaron el uso de esta estrategia. ${ }^{6,12}$

Una revisión encontró resultados semejantes en cuanto a la tendencia en la realización de estudios cuantitativos, como también la falta de investigación sobre la formación de los docentes para el uso de la simulación. ${ }^{13}$ En el contexto de la simulación clínica, muchos son los actores involucrados y cada uno tiene una parte de responsabilidad para que la estrategia se haga efectiva. Además de los profesores y de los estudiantes, se puede pensar en investigar a los pacientes simulados, aquéllos que son cuidados por profesionales formados con el uso de esta estrategia de enseñanza, los mismos profesionales egresados de esas escuelas $y$, por qué no también, los nuevos docentes que se formaron con simulación y actualmente enseñan en este medio.

La concentración de estudios en el sudeste se justifica por ser una región más desarrollada, con un número mayor de universidades renombradas que invirtieron en los últimos años en modernos laboratorios de simulación.

Varios estudios señalaron la contribución de la simulación para la retención de conocimientos. Una revisión sistemática identificó que la simulación es útil en la creación de un ambiente de aprendizaje que contribuya para el conocimiento, las habilidades, la seguridad y la confianza; sin embargo, existe una laguna en la literatura sobre la transferencia de esos resultados a los escenarios clínicos. ${ }^{14,15}$

La satisfacción de los alumnos en el uso de la simulación clínica también es relatada en otras investigaciones. ${ }^{16,17}$ Se trata de un elemento importante en la evaluación de la efectividad de una estrategia de enseñanza, pues indica mayor involucro y motivación del estudiante para el aprendizaje. ${ }^{18}$

Incluso están disponibles algunos instrumentos validados para su uso en la simulación, que buscan evaluar la satisfacción del estudiante, como la Escala de Satisfacción con las Experiencias Clínicas Simuladas-ESECS ${ }^{18}$ validada al portugués y la Simulation Experience Scale$\mathrm{SSES},{ }^{19}$ entre otras existentes en lengua inglesa.

Se nota cada vez más la necesidad de instrumentos que auxilien el monitoreo del aprendizaje por simulación, así como la formación docente para el uso de esta estrategia metodológica.

La metodología de la simulación y cada una de sus etapas han sido exploradas buscando comprobar su eficiencia. Hay estudios que han comparado su uso con la aplicación de otras estrategias de enseñanza. Una revisión sistemática evaluó 15 ensayos clínicos aleatorizados y concluyó que la simulación parece ser una efectiva estrategia para mejorar las habilidades de los enfermeros en comparación con otras estrategias de enseñanza. Sin embargo, la calidad de las evidencias fue clasificada como baja, lo que indica incertidumbre. ${ }^{20}$

\section{CONCLUSIÓN}

La investigación sobre simulación clínica en Brasil es reciente, tuvo un crecimiento expresivo en el año 2017 y se concentra en evaluar la eficacia del método en la retención de contenidos específicos.

La enfermería está liderando las encuestas sobre simulación y el abordaje cualitativo es incipiente. Poco se ha discutido sobre la parte pedagógica del método y no aparecen investigaciones sobre la interdisciplinariedad. Ese estudio tiene por limitación trabajar solamente con investigaciones provenientes de tesis y disertaciones de doctorado y máster. Se pueden desarrollar otros trabajos que busquen identificar y caracterizar las producciones brasileñas en la graduación, especialización, residencia, trabajos presentados en eventos de simulación clínica, entre otros espacios de desarrollo y compartición de conocimientos.

\section{Agradecimientos}

El presente trabajo contó con el apoyo de la Coordinación de Perfeccionamiento de Personal de Nivel Superior-Brasil (CAPES)-Código de Financiación 001.

De igual manera, con el apoyo del Consejo Nacional de Desarrollo Científico y Tecnológico-Brasil (CNPq).

\section{REFERENCIAS}

1. Jones F, Passos-Neto CE, Braghiroli OFM. Simulação em educação médica: breve histórico e metodologia. PPCR. 2015, 1 (2): 56-63. 
2. Rosen KR. The history of medical simulation. J Crit Care. 2008; 23 (2): 157-166.

3. Nickerson M, Pollard M. Mrs. Chase and her descendants: a historical view of simulation. Creat Nurs. 2010; 16 (3): 101-105.

4. Herrmann EK. Remembering Mrs. Chase. Before there were Smart Hospitals and Sim-Men, there was "Mrs. Chase". Imprint. 2008; 55 (2): 52-55.

5. Mora-Quirós S, de Oliveira-Vargas MA. Simulação clínica: uma estratégia que articula práticas de ensino e pesquisa em enfermagem. Texto \& Contexto Enfermagem. 2014; 23 (4): 813-814.

6. Vieira RQ, Caverni LMR. Manequim de simulação humana no laboratório de enfermagem: uma revisão de literatura. Hist Enferm Rev Eletrônica. 2011; 2 (1): 105-120.

7. Bland AJ, Topping A, Wood B. A concept analysis of simulation as a learning strategy in the education of undergraduate nursing students. Nurse Educ Today. 2011; 31 (7): 664-670.

8. Araújo ALLS, Quilici AP. O que é simulação e por que simular. En: Quilici AP, Abrão K, Timerman S, Gutierrez F. Simulação clínica: do conceito à aplicabilidade. São Paulo: Editora Atheneu; 2012. pp. 1-16.

9. Amaya-Afanador A. Simulación clínica: “aproximación pedagógica de la simulación clínica". Universitas Médica. 2010; 51 (2): 204-211.

10. de Souza-Teixeira CR, Kusumota L, Merizio-Martins Braga FT, Pirani-Gaioso V, Benedita dos Santos C, de Sousa e Silva VL, et al. O uso de simulador no ensino de avaliação clínica em enfermagem. Texto Contexto Enferm. 2011; 20: 187-193.

11. Sasso-Mendes KD, de Campos-Pereira Silveira RC, Galvão CM. Revisão integrativa: método de pesquisa para a incorporação de evidências na saúde e na enfermagem. Texto Contexto Enferm. 2008; 17 (4): 758-764.

12. Gomes CO. Entre orquídeas e girassóis: o laboratório de enfermagem na visão de estudantes. [Dissertação]. Natal: Centro de Ciências da Saúde da Universidade Federal do Rio Grande do Norte. 2004. 106 p. Mestrado em Enfermagem. pp. 28, 39.
13. Jorge, BM, Almeida, RGS, Souza Jr VD. Tendências atuais na investigação em simulação. In: Martins JCA, Mazzo A, Mendes IAC, Rodrigues MA. A simulação no ensino de enfermagem. Ribeirão Preto: SOBRACEn; 2014.

14. Norman J. Systematic review of the literature on simulation in nursing education. ABNF J. 2012; 23 (2): 24-28.

15. Zapko KA, Ferranto MLG, Blasiman R, Shelestak D. Evaluating best educational practices, student satisfaction, and self-confidence in simulation: a descriptive study. Nurse Educ Today. 2018; 60: 2834.

16. Woodruff K, O’Neill SP, Walton-Moss BJ. Exploring APN students' perceptions, self-confidence, and satisfaction with clinical simulation. Nurs Educ Perspect. 2017; 38 (6): 347-349.

17. McRae ME, Chan A, Hulett R, Lee AJ, Coleman $B$. The effectiveness of and satisfaction with high-fidelity simulation to teach cardiac surgical resuscitation skills to nurses. Intensive Crit Care Nurs. 2017; 40: 64-69.

18. Negrão-Baptista RC, Amado-Martins JC, Carneiro Ribeiro Pereira MF, Mazzo A. Satisfação dos estudantes com as experiências clínicas simuladas: validação de escala de avaliação. Rev Lat Am Enfermagem. 2014; 22 (5): 709-715.

19. Williams B, Dousek S. The satisfaction with simulation experience scale (SSES): a validation study. J Nurs Educ Pract. 2012; 2 (3): 74-80.

20. Hegland PA, Aarlie H, Strømme H, Jamtvedt G. Simulation-based training for nurses: Systematic review and meta-analysis. Nurse Educ Today. 2017; 54: 6-20.

Correspondencia:

Saionara Nunes de Oliveira

Rua José Ferminio Novaes 719, apto. 203,

Bairro Kobrasol, São José,

Santa Catarina, Brasil. CEP 88102080

Telefone: +5548999425620

E-mail: saionaranunes@gmail.com 